\title{
Generalized Extreme Value Distribution Models for the Assessment of Seasonal Wind Energy Potential of Debuncha, Cameroon
}

\author{
Nkongho Ayuketang Arreyndip ${ }^{1,2,3}$ and Ebobenow Joseph ${ }^{1}$ \\ ${ }^{1}$ Department of Physics and Applied Physics, University of Buea, Buea, Cameroon \\ ${ }^{2}$ African Institute for Mathematical Science, 608 Limbe, Cameroon \\ ${ }^{3}$ Polytechnic, Saint Jerome Catholic University Institute of Douala, Akwa, 5949 Douala, Cameroon \\ Correspondence should be addressed to Nkongho Ayuketang Arreyndip; ayuketang@aims-cameroon.org
}

Received 17 June 2016; Revised 16 August 2016; Accepted 3 October 2016

Academic Editor: Pallav Purohit

Copyright (C) 2016 N. Ayuketang Arreyndip and E. Joseph. This is an open access article distributed under the Creative Commons Attribution License, which permits unrestricted use, distribution, and reproduction in any medium, provided the original work is properly cited.

\begin{abstract}
The method of generalized extreme value family of distributions (Weibull, Gumbel, and Frechet) is employed for the first time to assess the wind energy potential of Debuncha, South-West Cameroon, and to study the variation of energy over the seasons on this site. The 29-year (1983-2013) average daily wind speed data over Debuncha due to missing values in the years 1992 and 1994 is gotten from NASA satellite data through the RETScreen software tool provided by CANMET Canada. The data is partitioned into min-monthly, mean-monthly, and max-monthly data and fitted using maximum likelihood method to the two-parameter Weibull, Gumbel, and Frechet distributions for the purpose of determining the best fit to be used for assessing the wind energy potential on this site. The respective shape and scale parameters are estimated. By making use of the $P$ values of the Kolmogorov-Smirnov statistic (K-S) and the standard error (s.e) analysis, the results show that the Frechet distribution best fits the min-monthly, meanmonthly, and max-monthly data compared to the Weibull and Gumbel distributions. Wind speed distributions and wind power densities of both the wet and dry seasons are compared. The results show that the wind power density of the wet season was higher than in the dry season. The wind speeds at this site seem quite low; maximum wind speeds are listed as between $3.1 \mathrm{and} 4.2 \mathrm{~m} / \mathrm{s}$, which is below the cut-in wind speed of many modern turbines $(6-10 \mathrm{~m} / \mathrm{s})$. However, we recommend the installation of low cut-in wind turbines like the Savonius or Aircon $(10 \mathrm{KW})$ for stand-alone low energy need.
\end{abstract}

\section{Introduction}

Cameroon which is also in the list of fast growing economies in Africa has experienced a very fast growth rate in the past two decades with the Government investing heavily on industrialization. Statistics has shown that the number of heavy and light industries has doubled since the early 90s. The population statistics has significantly increased from 15 million inhabitants in the 80 s to about 21 million inhabitants. These are the two main concepts that plunge an economy energy sector into serious challenges like energy shortages and distribution. The concept of energy shortage is a global issue as many world economies now have embarked on alternative renewable energy sources to meet their energy demand. Also faced with the crisis of global warming, the Paris summit with Cameroon represented by its head of state was to tackle this issue with most world economies accepting the $1.5^{\circ} \mathrm{C}$ rise in temperature and shifting their energy production from fossil fuels combustion to renewable energy enhancement. Cameroon is known for its enormous energy potentials. There is abundance of heavy water falls in the south to harness hydroelectric power, high solar intensities especially the northern regions for solar power enhancement, and wind power in its coastal cities for wind energy enhancement. These sectors are underdeveloped and the country solely relies on hydroelectric power to meet its energy demand with most of these rivers seasonal. Therefore it is worthwhile looking for other alternative sources of energy like wind and 
solar energy. To successfully harness wind power at a site, a wind energy assessment needs to be carried out which usually results in analysing the wind speed data at the particular site and also studying the topographic nature of the site.

Many researchers have applied statistical techniques to assess the wind energy potential of a particular site or sites like the commonly used Weibull statistics and others like the lognormal and Rayleigh distributions. For example, Fagbenle et al. [1] applied the Weibull statistics and other statistical methods to assess the wind energy potentials of two sites in north-east Nigeria and found that Maiduguri was the better of the sites as compared to Potiskum in terms of monthly and seasonal variation of mean wind speed, but they both can be suitable for stand-alone and medium scale wind power generation. Oyedepo et al. [2] also applied the Weibull statistics to analyse wind speed data and wind energy potential in three selected locations (Enugu, Owerri, and Onitsha) in south-east Nigeria, where they found that Enugu was the best site with high wind energy potential. Nikolai Nawri et al. applied the downscaling simulations technique performed with the Weather Research and Forecasting (WRF) model to determine the large-scale wind energy potential of Iceland with local wind speed distributions represented by Weibull statistics. They found that, in addition to seasonal and spatial variability, differences in average wind speed and power density also exist for different wind directions. Kollu et al. [3] applied the three mixture probability density functions: Weibull-extreme value distribution (GEV), Weibulllognormal, and GEV-lognormal which were not tried before to describe (model) wind speed characteristics. By making use of statistical parameters such as maximum error in the Kolmogorov-Smirnov test, root mean square error, Chisquare error, coefficient of determination, and power density error as judgement criteria to assess the fitness of the probability density functions, they found that the WeibullGEV PDF is able to describe unimodal as well as bimodal wind distributions accurately whereas GEV-lognormal PDF is able to describe familiar bell-shaped unimodal distribution well. They also found that mixture probability functions are better alternatives to conventional Weibull, two-component mixture Weibull, gamma, and lognormal PDFs to describe wind speed characteristics.

Most of the results in this area show that the Weibull distribution is the best distribution to mathematically model the wind energy potential of a particular site. So in this paper, our focus and analysis are based on the fact that, being a member of the generalized extreme value class of distributions also known as generalized extreme value type III distribution, it is worthwhile also to study the other class of distributions (Gumbel (type I), Frechet (type II)) in the same family to know if there is another distribution that can best be used to describe the wind energy potential of a site.

1.1. Geophysical Description of Debuncha. Debuncha, being known as the rainiest place in central and west Africa, is a small Cameroonian village on the western coast of the city of Limbe at the windward side of mount Cameroon. Some wind energy experts have assessed this site and it has been found suitable for the installation of an on-shore wind turbine

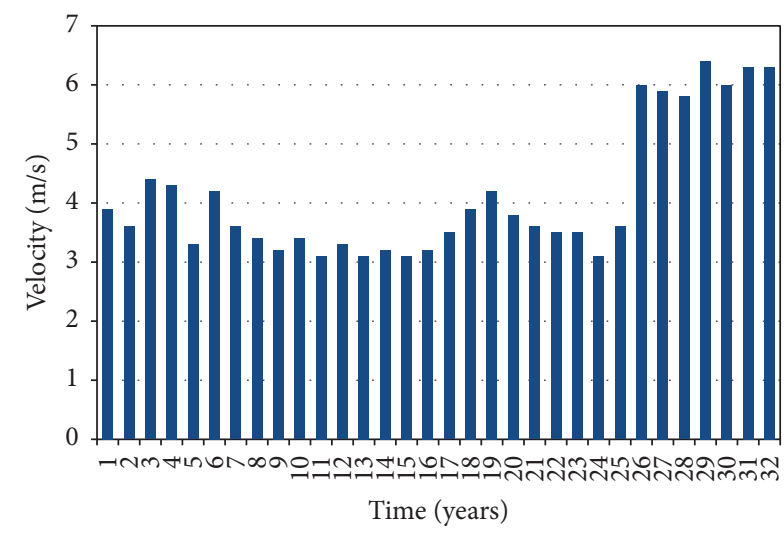

FIgURE 1: Annual maximum wind speed for Debuncha.

because of its topographic structure and also because of its accessibility. So our main aim in this work is to select in the family of generalized extreme value class of distributions which distribution is best suited to use for wind power assessment and modelling for the feature installation of an on-shore wind turbine, study the conditions under which the twoparameter Weibull distribution which has so far been termed the best statistical distribution for wind power modelling and assessment can best be used, and finally to study the wind power variation over the seasons on this site.

The paper is arranged in three sections; in Section 2, we present the mathematical models of the underlying theory and statistically analyse the data set. Section 3 is dedicated to discussing the results of our findings and we end with a conclusion in Section 4.

\section{Data and Method}

2.1. Raw Data Analysis. The data provided to us for analysis is NASA satellite daily average wind data over Debuncha on the western coast of Limbe, Cameroon, with longitude 9.2149, latitude 4.0242, and elevation of about $36 \mathrm{~m}$ above sea level. This data contains 29 (1983-2013) years of mean daily data with missing values in the years 1992 and 1994 . Hence these years were considered as outliers. The data was sorted out into monthly means (mean-monthly), monthly minimums (minmonthly), and monthly maximums (max-monthly) for the 29 years.

The second analysis deals with comparing the wind energy potentials of the two main seasons that exist in Cameroon which are the wet and dry seasons. The wet seasons usually run from May to September, while the dry season usually runs from November to March. In April and October there usually occur the short Springs and Autumn seasons, respectively. So they were not included in the analysis. The data is again partitioned into the five months' dry season (November to March) and five months' wet season (May to September) for analysis over the 29 years' period. The yearly maximum wind speeds over this area for the 29 years' period are shown in Figure 1 with missing data in 1992 and 1994. The min-monthly, mean-monthly, and max-monthly wind distributions are also shown in Figures 2, 3, and 4. 


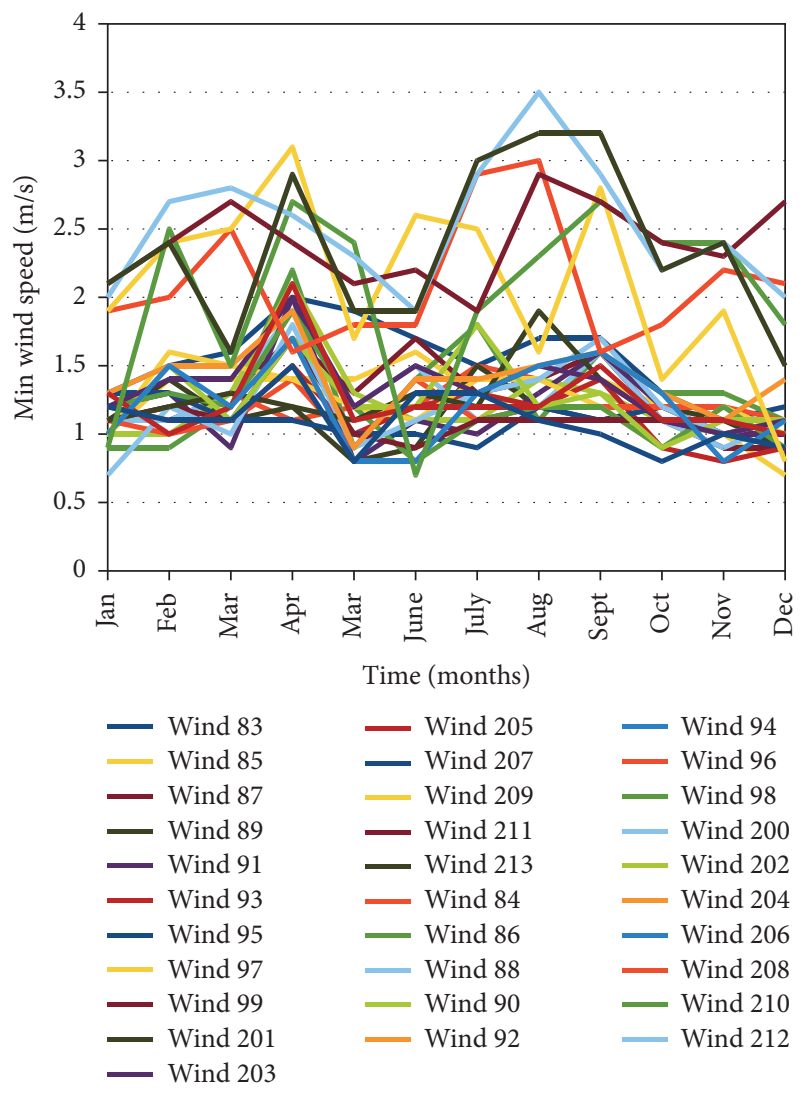

FIGURE 2: Plot of monthly minimums wind speed distribution over Debuncha for 31 years' period.

2.2. Mathematical Analysis. We will start this section by reviewing the cumulative distribution function of the threeparameter generalized extreme value (GEV) distribution given by

$$
F(x)=\exp \left\{-\left[1+\xi\left(\frac{x-\mu}{\sigma}\right)\right]^{-1 / \xi}\right\} .
$$

Define, for $\{x: 1+\xi(x-\mu) / \sigma>0\},-\infty<\mu<\infty$, $\sigma>0$, and $-\infty<\xi<\infty$, where $\mu$ is the location parameter, $\xi$ is the shape parameter, and $\sigma$ is the scale parameter $[4,5]$. There are three classes of the generalized extreme value family of distribution. Their differences depend only on the value of the shape parameter $\xi$.

We have the Gumbel distribution with $\xi=0$ with probability density function, cumulative probability distribution, and quantiles given by

$$
\begin{aligned}
& f(x)=\frac{1}{\sigma} \exp [z] \exp [-\exp (z)] \\
& F(x)=1-\exp (-\exp (z)) \\
& Q(x)=\mu-\sigma \log (-\log (x))
\end{aligned}
$$

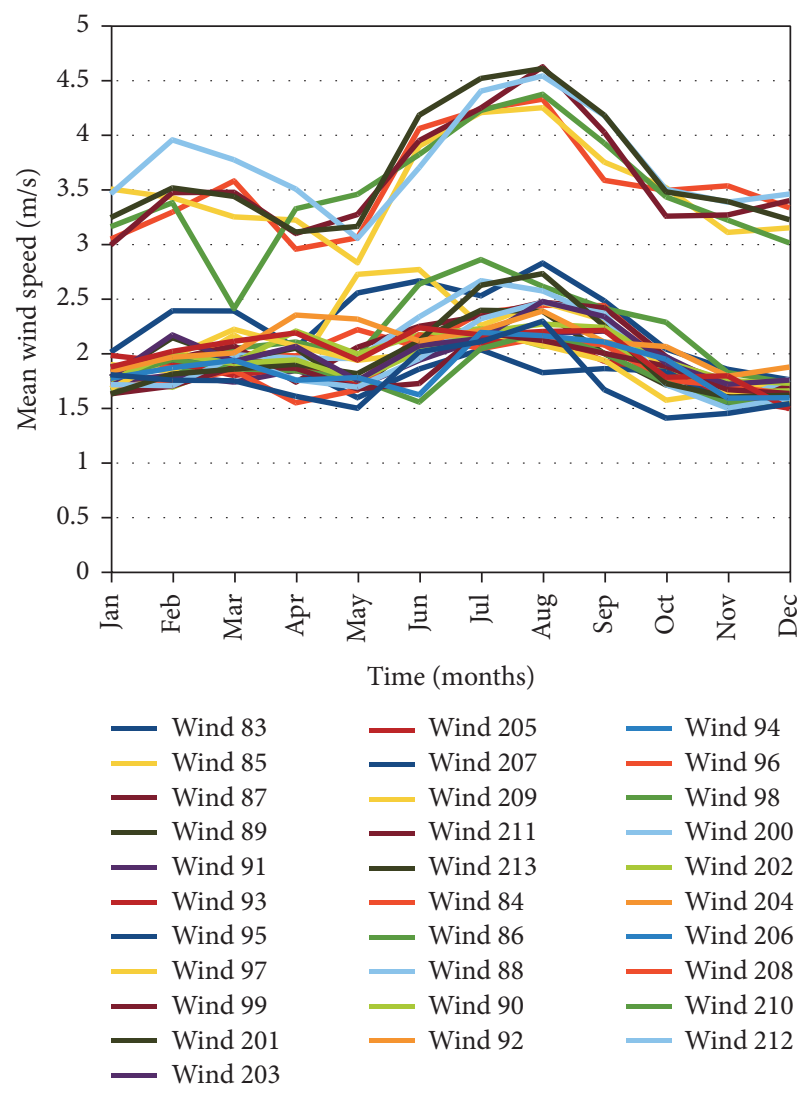

FIGURE 3: Plot of monthly average wind speed distribution over Debuncha for 31 years' period.

where

$$
z \equiv \frac{x-\mu}{\sigma} .
$$

For $\xi<0$ we have the Weibull distribution with probability density function, cumulative probability distribution, and quantile distribution given by

$$
\begin{aligned}
& f(x)=\left(\frac{\xi}{\sigma}\right)\left(\frac{x}{\sigma}\right)^{\xi-1} \exp \left[-\left(\frac{x}{\sigma}\right)^{\xi}\right] \\
& F(x)=1-\exp \left(-\left(\frac{x}{\sigma}\right)^{\xi}\right) \\
& Q(x)=\sigma[-\log (1-x)]^{1 / \xi} .
\end{aligned}
$$

For $\xi>0$, we have the Freshet class of distribution with cumulative probability distribution given by

$$
\begin{aligned}
& f(x)=\frac{\xi}{\sigma}\left(\frac{\sigma}{x}\right)^{\xi+1} \exp \left[\left(\frac{\sigma}{x}\right)^{\xi}\right] \\
& F(x)=\exp \left(-\left(\frac{\sigma}{x}\right)^{\xi}\right) \\
& Q(x)=\frac{\sigma}{(-\log (x))^{(1 / \xi)}}
\end{aligned}
$$




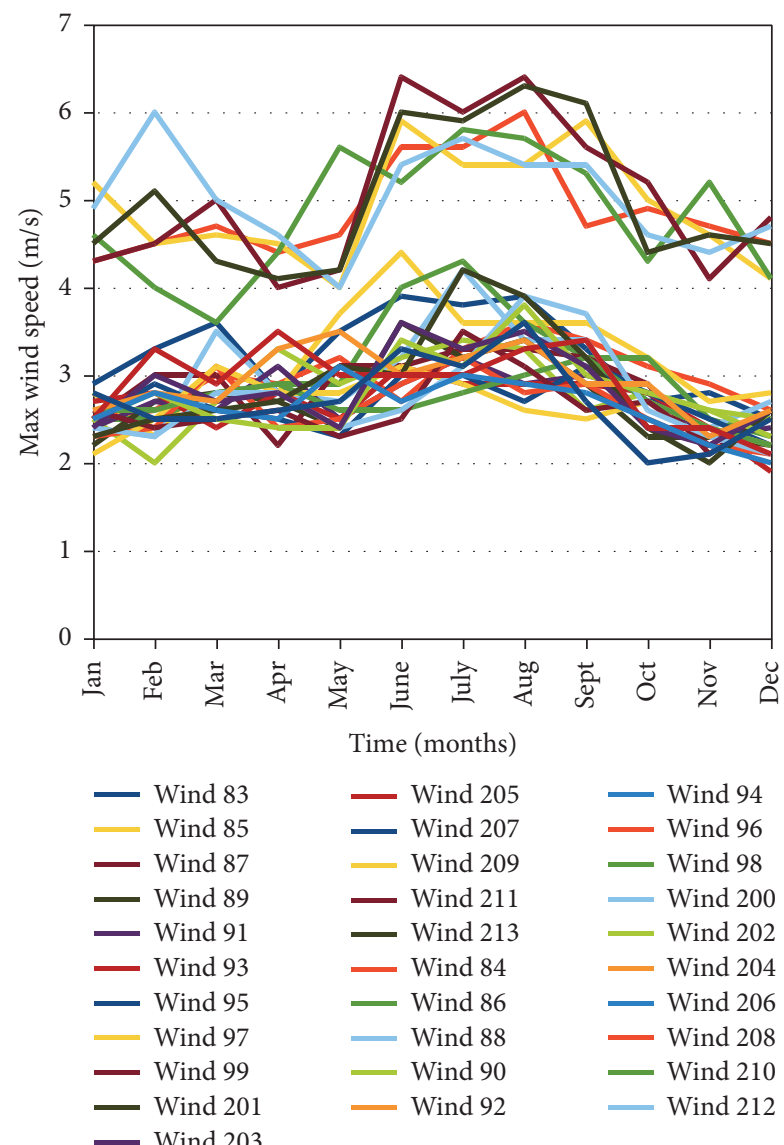

FIGURE 4: Plot of monthly maximums wind speed distribution over Debuncha for 31 years' period.

Now using the Weibull distribution, the mean values of the wind speed $x_{m}$ and standard deviation $\nu$ defined in terms of the Weibull parameters $\xi$ and $\sigma$ are given as

$$
x_{m}=\sigma \Gamma\left(1+\frac{1}{\xi}\right),
$$

where $\Gamma()$ is the gamma function, and

$$
\nu=\sqrt{\sigma^{2}\left[\Gamma\left(1+\frac{2}{\xi}\right)-\left[\Gamma\left(1+\frac{1}{\xi}\right)\right]^{2}\right]} .
$$

2.3. Mean Power and Energy Densities. When assessing the wind energy potential of a site, there are two wind speeds that are of interest and must therefore be taken seriously into consideration. These are the most probable wind speed $v_{\mathrm{mp}}$ and the wind speed carrying maximum energy $v_{E \max }$. They are given by the expressions $[6,7]$

$$
\begin{aligned}
v_{\mathrm{mp}} & =\sigma\left(\frac{\xi-1}{\xi}\right)^{(1 / \xi)}, \\
v_{E \max } & =\sigma\left(\frac{\xi+2}{\xi}\right)^{(1 / \xi)} .
\end{aligned}
$$

Theoretically, the mean power density is proportional to the cube of the mean velocity given by

$$
P_{D}=\frac{P\left(v_{m}\right)}{A}=\frac{1}{2} \rho v_{m}^{3} .
$$

It can also be calculated from the Weibull probability density function given by

$$
P_{D}=\frac{P(v)}{A}=\frac{1}{2} \rho \sigma^{3} \Gamma\left(1+\frac{3}{\xi}\right)
$$

and the energy density is given by

$$
E_{D}=\frac{1}{2} \rho \sigma^{3} \Gamma\left(1+\frac{3}{\xi}\right) T,
$$

where $P_{D}$ is the power density, $E_{D}$ the energy density, $P(v)$ wind power, $A$ cross-sectional area of rotor, $\rho$ the air density assumed here to take the value $1.225, c$ scale parameter, $\xi$ shape parameter, $T$ period, and $\Gamma()$ the gamma function $[1,6,7]$.

The data set over the operating conditions (min-monthly, mean-monthly, and max-monthly) is fitted into the GEV class of distribution in $\mathrm{R}$ using the R-package "fitdistrplus" using the maximum goodness of fit and the parameters are estimated using the method of maximum likelihood which is given by $[8,9]$

$$
\xi=\left[\frac{\sum_{i=1}^{n} v_{i}^{\xi} \ln \left(v_{i}\right)}{\sum_{i=1}^{n} v_{i}^{\xi}}-\frac{\sum_{i=1}^{n} \ln \left(v_{i}\right)}{n}\right]^{-1},
$$

where $v_{i}$ is the wind speed in time step $i$ and $n$ is the number of data points.

The scale parameter is given by

$$
\sigma=\left[\frac{1}{n} \sum_{i=1}^{n} v_{i}^{\xi}\right]^{1 / \xi}
$$

\section{Results and Discussion}

The results of the method applied here are presented in Figures 2-9 and Table 1.

The parameters of the Weibull, Gumbel, and Freshet distributions are estimated using the method of maximum likelihood and are displayed in Table 1.

Figure 1 shows the yearly maximum wind speed distributions for the 29 years' period. We see that there is an increase in the wind speed from where it usually fluctuates from $3.1 \mathrm{~m} / \mathrm{s}$ to $4.2 \mathrm{~m} / \mathrm{s}$ from 1983 to 2006 to about $6.0 \mathrm{~m} / \mathrm{s}$ starting from the year 2007 to 2013 . This might be as a result of the changes in the pressure gradient and local weather conditions of the place.

Figures 2, 3, and 4 show that one of the most windy months is August which is usually at the heart of the wet season, and we also note from these figures that the month with the least wind speed is the month of December which is in the dry season and the highest year with a high average recorded wind speed is 2013. 
TABLE 1: Data summary statistics and parameter estimates.

\begin{tabular}{|c|c|c|c|}
\hline Distributions & $\begin{array}{l}\text { Min wind } \\
\text { speed data }\end{array}$ & $\begin{array}{c}\text { Average } \\
\text { wind speed } \\
\text { data }\end{array}$ & $\begin{array}{l}\text { Max wind } \\
\text { speed data }\end{array}$ \\
\hline \multicolumn{4}{|l|}{ Weibull } \\
\hline$k$ & 2.76 & 3.13 & 3.29 \\
\hline s.e & 0.343 & 0.12 & 0.125 \\
\hline$c(\mathrm{~m} / \mathrm{s})$ & 1.62 & 2.56 & 3.63 \\
\hline s.e & 1.00 & 0.05 & 0.063 \\
\hline$P$ values of K-S & 0.175 & 0.182 & 0.172 \\
\hline \multicolumn{4}{|l|}{ Gumbel } \\
\hline$k$ & 1.22 & 1.98 & 2.833 \\
\hline s.e & 0.019 & 0.026 & 0.037 \\
\hline$c(\mathrm{~m} / \mathrm{s})$ & 0.35 & 0.47 & 0.665 \\
\hline s.e & 0.016 & 0.021 & 0.03 \\
\hline$P$ values of K-S & 0.149 & 0.108 & 0.121 \\
\hline \multicolumn{4}{|l|}{ Frechet } \\
\hline$k$ & 4.88 & 4.88 & 4.73 \\
\hline s.e & 0.22 & 0.23 & 0.20 \\
\hline$c(\mathrm{~m} / \mathrm{s})$ & 1.93 & 1.93 & 2.76 \\
\hline s.e & 0.022 & 0.022 & 0.033 \\
\hline$P$ values of $\mathrm{K}-\mathrm{S}$ & 0.109 & 0.083 & 0.074 \\
\hline \multicolumn{4}{|c|}{ Descriptive statistics } \\
\hline Mean (m/s) & 1.44 & 2.29 & 3.26 \\
\hline s.d & 0.54 & 0.74 & 1.0 \\
\hline $\operatorname{Min}(\mathrm{m} / \mathrm{s})$ & 0.7 & 1.4 & 1.9 \\
\hline $\operatorname{Max}(\mathrm{m} / \mathrm{s})$ & 3.5 & 4.62 & 6.4 \\
\hline Skew & 1.39 & 1.36 & 1.26 \\
\hline Kurtosis & 1.41 & 0.9 & 0.79 \\
\hline s.e & 0.03 & 0.04 & 0.05 \\
\hline
\end{tabular}

Figures 5 and 6 display the mean wind speeds and wind power densities of the dry and wet seasons. Considering the mean data, the mean and maximum wind velocities in the dry season were calculated to be $2.61 \mathrm{~m} / \mathrm{s}$ and $3.22 \mathrm{~m} / \mathrm{s}$, respectively, while those in the wet season are $3.36 \mathrm{~m} / \mathrm{s}$ and $4.17 \mathrm{~m} / \mathrm{s}$, respectively. Hence we see that, during the wet season, there is a possibility of generating higher power from a wind turbine on this area than in the dry season.

From Figures 7, 8, and 9, we fit the monthly minimum, monthly average, and monthly maximums wind speed data into the generalized extreme value class of distributions. The parameter was estimated using the method of maximum likelihood and presented in Table 1. Taking into consideration the $P$ values of the Kolmogorov-Smirnov test statistic (K-S) and the standard error (s.e) analysis, we see that the Frechet distribution best fits with the data in all operating conditions (min, mean, and max) followed by the Gumbel and finally the Weibull distribution. Here, we also see that the density, $Q Q$, and cdf plots throughout the data ranges best fit with the Frechet distribution than the Weibull and Gumbel distributions. These figures also show that the monthly average

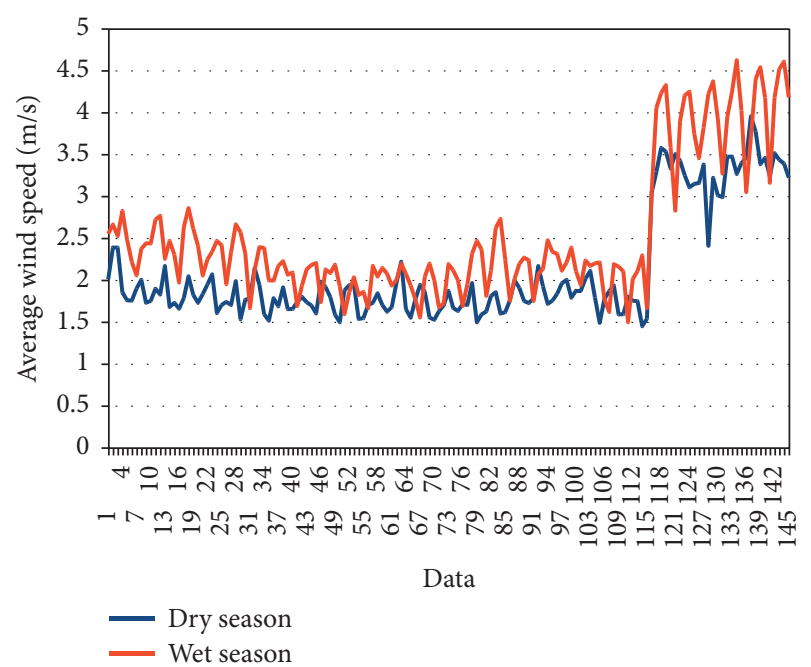

Figure 5: Mean wind speed distributions over the dry and wet seasons.

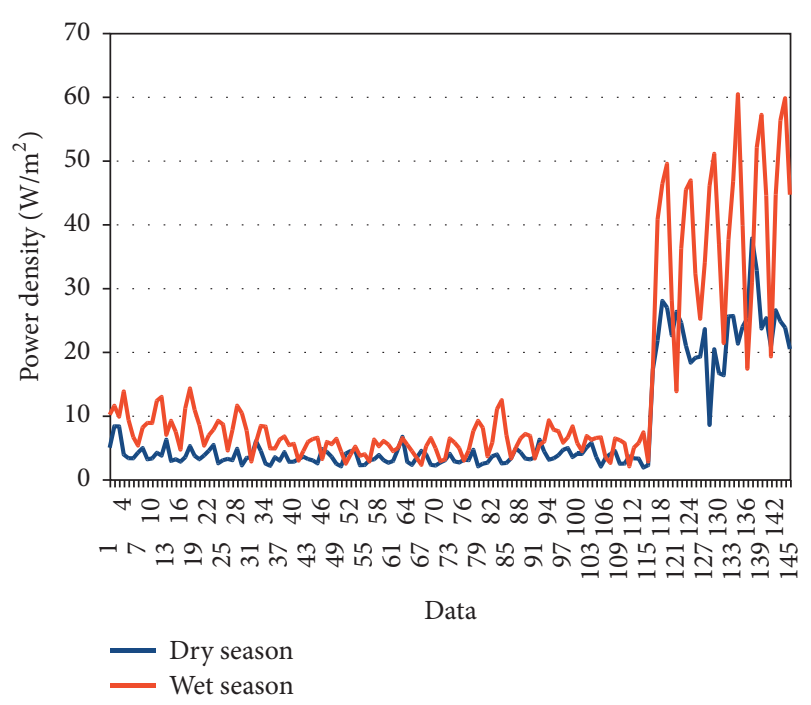

FIGURE 6: Mean wind power densities of both the dry and wet seasons.

wind speeds best fit the Weibull distribution as compared to the monthly minimums and the monthly maximums. This can also be confirmed by its lowest standard error and the $P$ values.

Table 1 also presents an overview of the descriptive statistics of the data set used. Here, we see that average monthly wind speeds over this site range from $1.4 \mathrm{~m} / \mathrm{s}$ to $4.62 \mathrm{~m} / \mathrm{s}$. Monthly average power density ranges from $5 \mathrm{~W} / \mathrm{m}^{2}$ to $60 \mathrm{~W} / \mathrm{m}^{2}$. These values are generally not encouraging for the installation of modern wind turbines.

\section{Conclusion}

To conclude, we have partitioned a 29 years' NASA satellite daily average wind speed data gotten through the RETScreen 

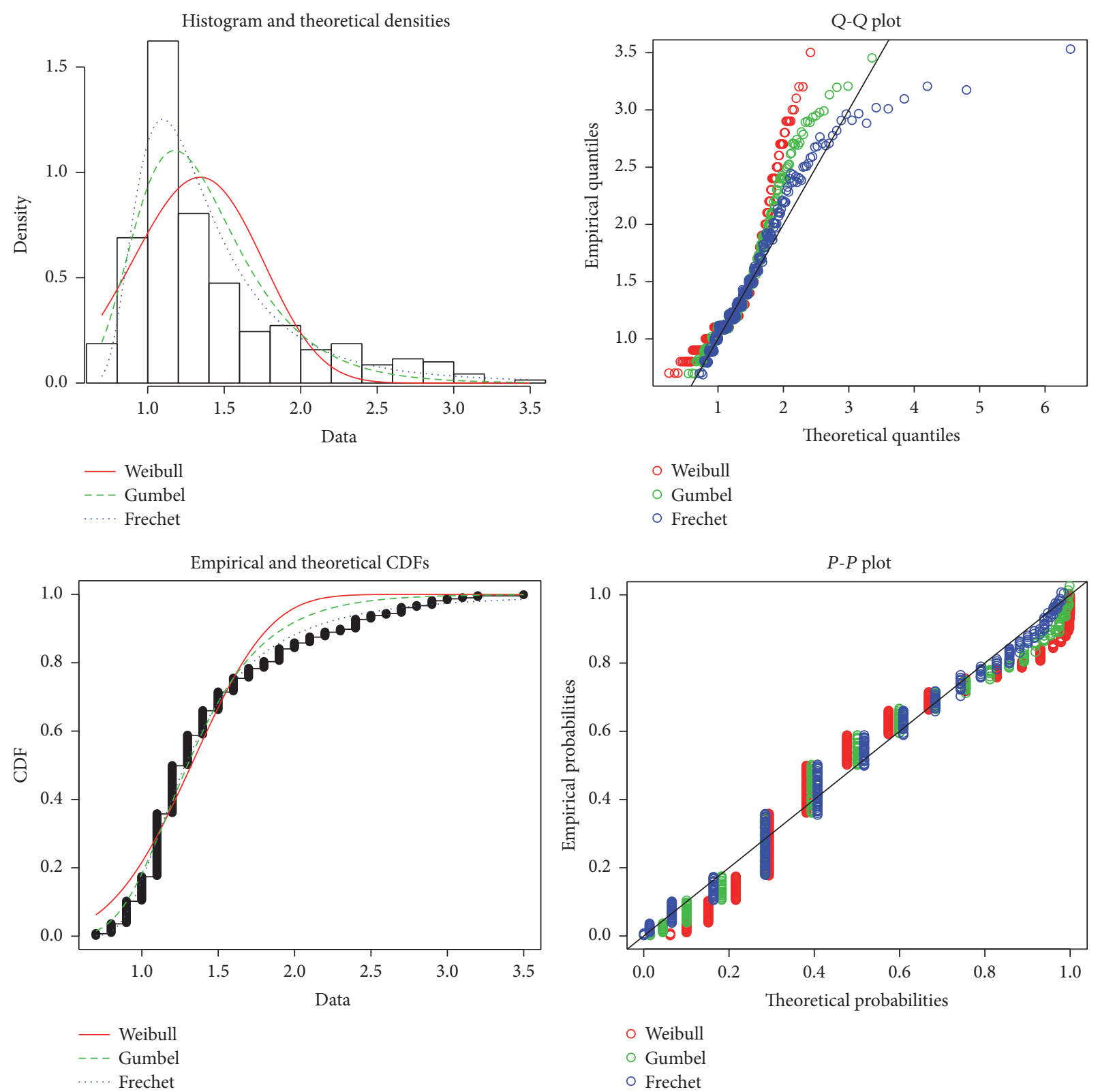

FIGURE 7: Monthly minimum wind speeds fitted to the GEV class of distributions.

software tool provided by CANMET Canada into monthly minimum, monthly average, and monthly maximum wind speeds and consequently fitted into the generalized extreme value class of distributions (Weibull, Frechet, and Gumbel) for the purpose of determining the best distribution for use to model the wind energy potential of Debuncha, Cameroon, and also to determine the condition under which the Weibull distribution which is so far the most used and recommended distribution for wind data analysis can be applied to the data. Using the Kolmogorov-Smirnov statistic and standard error analysis, the results here show that the Frechet distribution best fits the data in all operating conditions than the Gumbel and Weibull distributions. We have also seen that the monthly average wind speeds best fit the Weibull distribution as compared to the monthly minimums and monthly maximums. Hence, we conclude that when doing a Weibull analysis of the data on this site, the monthly average wind speeds should be used. The wind speed distributions of the dry and wet seasons together with the power densities over these seasons were analysed where we have seen that there is a greater wind speed and hence a higher power density in the wet season as compared to the dry season. The time series analysis of the yearly maximum wind speed data over the 29 years' period as shown in Figure 1 shows that there is an increasing trend in the wind speed for Debuncha from a maximum of $3.1 \mathrm{~m} / \mathrm{s}$ to $4.2 \mathrm{~m} / \mathrm{s}$ from 1983 to 2006 to above $6.0 \mathrm{~m} / \mathrm{s}$ in 2013 which 

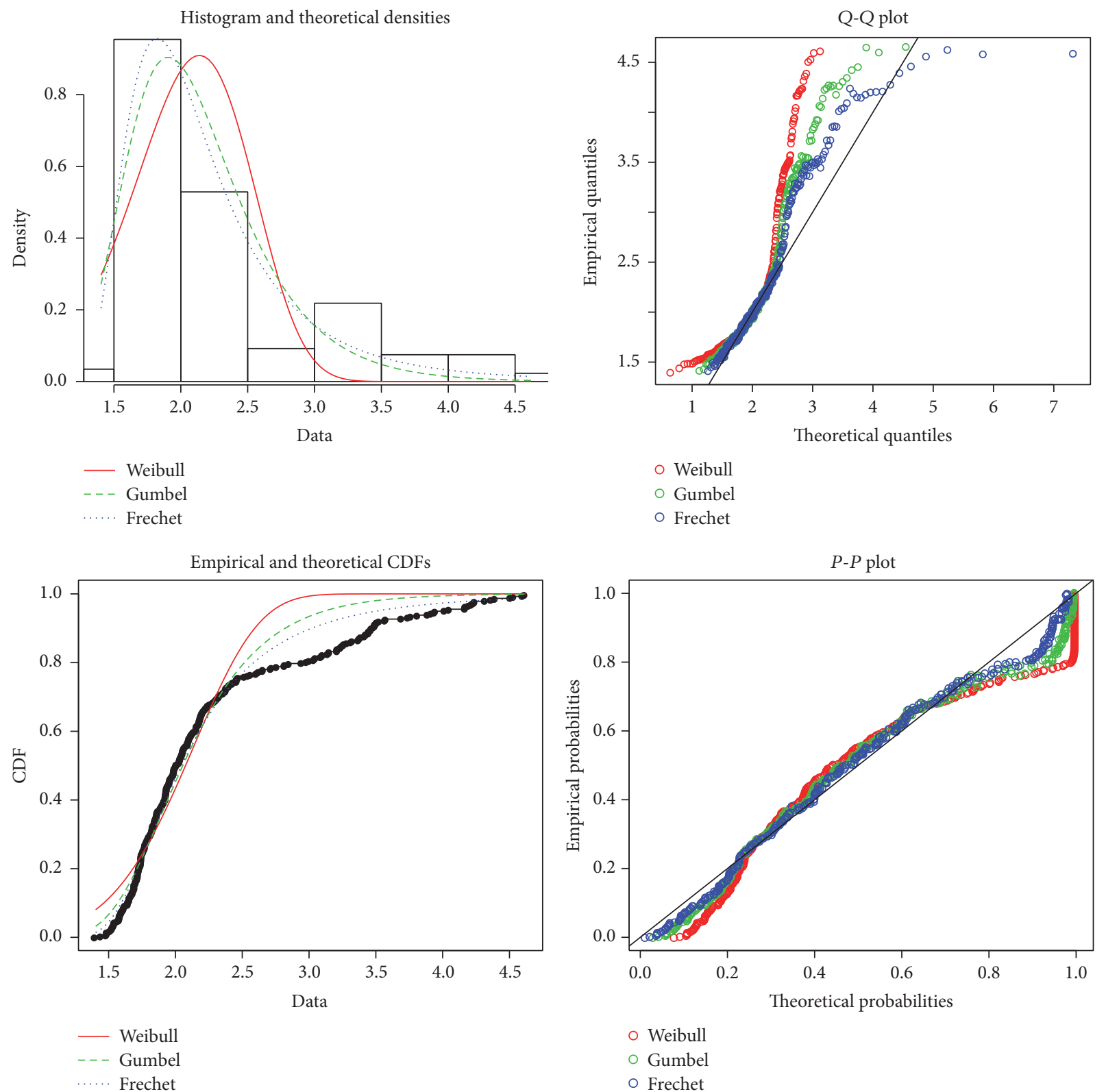

FIGURE 8: Monthly average wind speeds fitted to the GEV class of distributions.

shows an increase in the wind power potential at this site. Hence from small to medium scale power generation, this may be suitable.

Generally, the average wind speeds over this site are relatively low as compared to the cut-in wind speeds of most modern wind turbines which are usually between 6 and $10 \mathrm{~m} / \mathrm{s}$. Hence to generate wind energy on this site, a wind farm made of small wind turbines with low cut-in wind speed such as the AIRCON (HAWT $10 \mathrm{KW}$ ) or the Savonius wind turbine is recommended.

Wind resource studies typically focus on high resolution data $10 \mathrm{~min}$ to 1 -hour intervals. But this data and ground station data for the site are not available at the moment for analysis. Hence, in future, we will make sure we get in touch with high resolution data or ground station data from Cameroon meteorological department over this site. We will also be looking forward to designing more adaptive small wind turbines which can make use of the low wind speed over this site by concentrating on the aerodynamic structure, reduced load, and air-foil design with high lift and low Reynold number.

\section{Competing Interests}

The authors declare that they have no competing interests. 

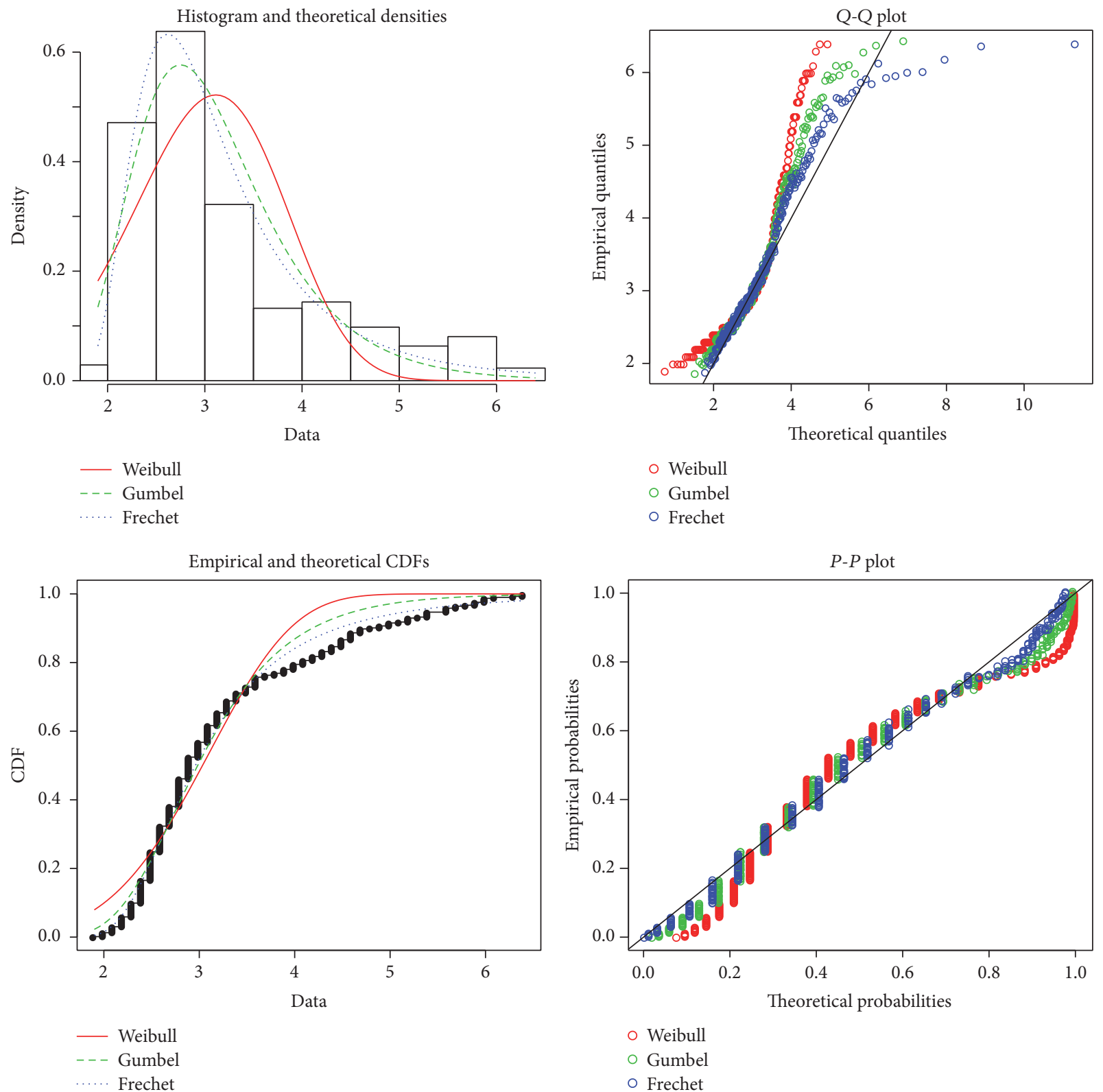

FIGURE 9: Monthly maximum wind speeds fitted to the GEV class of distributions.

\section{Acknowledgments}

This work was carried out with financial support from the Government of Canada's International Development Research Centre (IDRC) and within the framework of the AIMS Research for Africa Project. The authors also like to thank the National Aeronautics and Space Administration (NASA) and CANMET Canada for providing them with the data.

\section{References}

[1] R. O. Fagbenle, J. Katende, O. O. Ajayi, and J. O. Okeniyi, "Assessment of wind energy potential of two sites in North-East, Nigeria," Renewable Energy, vol. 36, no. 4, pp. 1277-1283, 2011.
[2] S. O. Oyedepo, M. S. Adaramola, and S. S. Paul, "Analysis of wind speed data and wind energy potential in three selected locations in south-east Nigeria," International Journal of Energy and Environmental Engineering, vol. 3, article 7, 2012.

[3] R. Kollu, S. R. Rayapudi, S. V. L. Narasimham, and K. M. Pakkurthi, "Mixture probability distribution functions to model wind speed distributions," International Journal of Energy and Environmental Engineering, vol. 3, article 27, 2012.

[4] E. S. Martins and J. R. Stedinger, "Generalized maximumlikelihood generalized extreme-value quantile estimators for hydrologic data," Water Resources Research, vol. 36, no. 3, pp. 737-744, 2000

[5] J. R. M. Hosking, "Algorithm AS 215: maximum-likelihood estimation of the parameters of the generalized extreme-value distribution," Journal of the Royal Statistical Society. Series C (Applied Statistics), vol. 34, no. 3, pp. 301-310, 1985. 
[6] B. Ozerdem and M. Turkeli, "An investigation of wind characteristics on the campus of Izmir Institute of Technology, Turkey," Renewable Energy, vol. 28, no. 7, pp. 1013-1027, 2003.

[7] J. A. Carta, P. Ramírez, and S. Velázquez, "A review of wind speed probability distributions used in wind energy analysis. Case studies in the Canary Islands," Renewable and Sustainable Energy Reviews, vol. 13, no. 5, pp. 933-955, 2009.

[8] D. C. Montgomery and G. C. Runger, Applied Statistics and Probability for Engineers, John Wiley \& Sons, New Jersey, NJ, USA, 3rd edition, 2003.

[9] E. K. Akpinar and S. Akpinar, "An assessment on seasonal analysis of wind energy characteristics and wind turbine characteristics," Energy Conversion and Management, vol. 46, no. 11-12, pp. 1848-1867, 2005. 

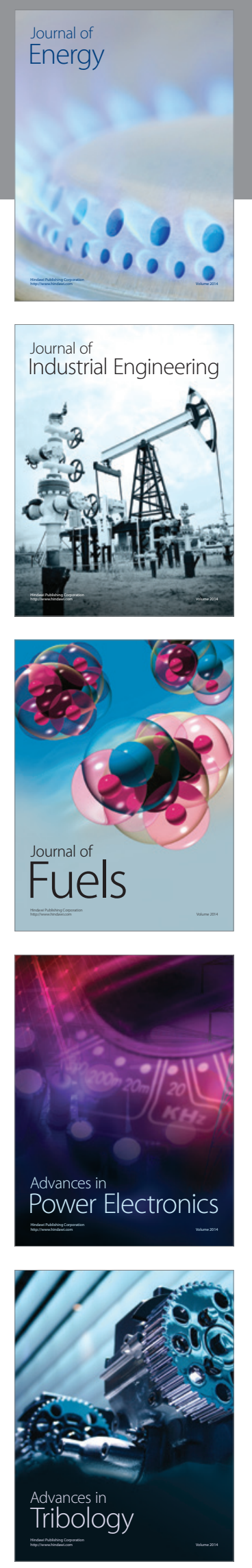
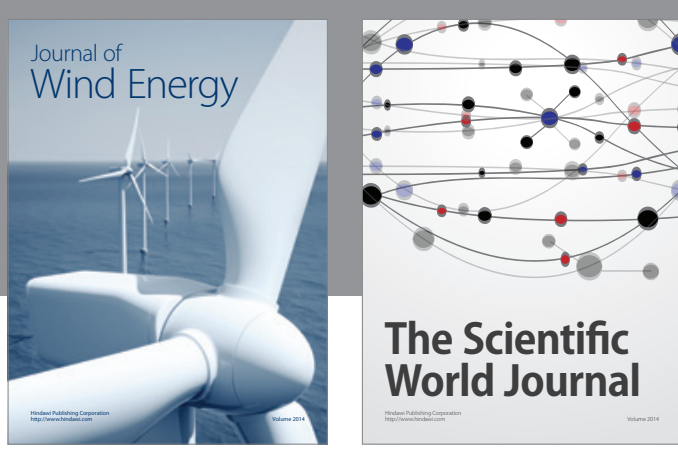

The Scientific World Journal
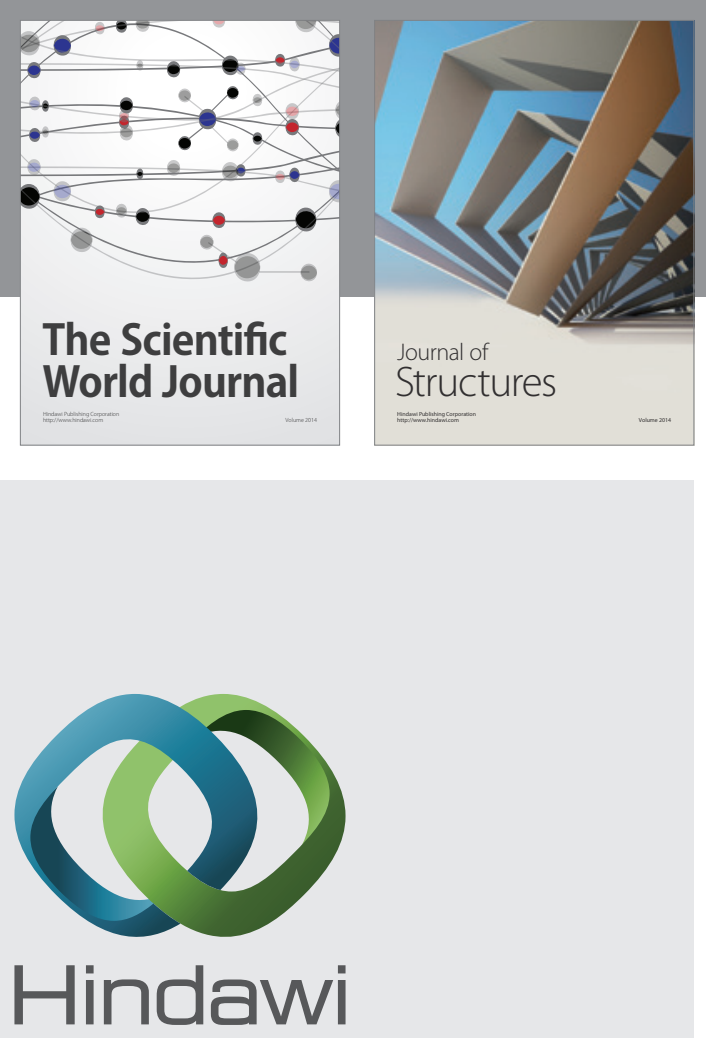

Submit your manuscripts at

http://www.hindawi.com
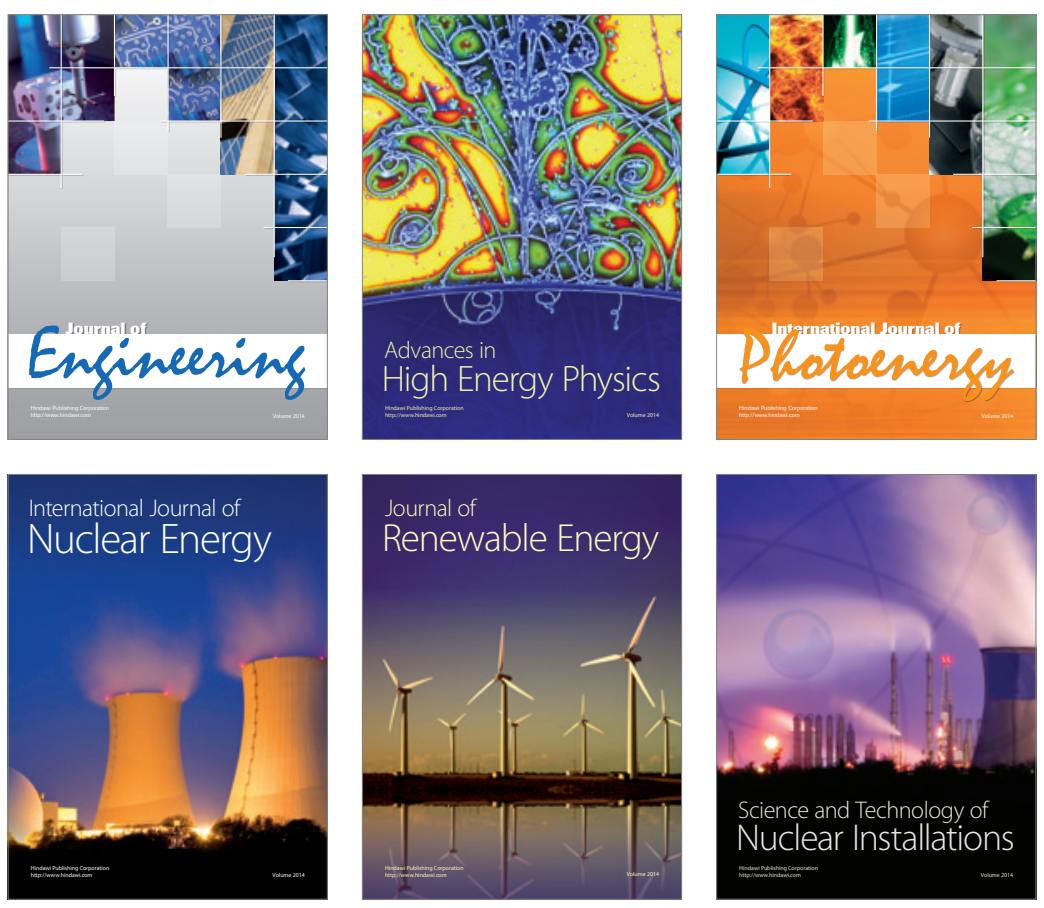
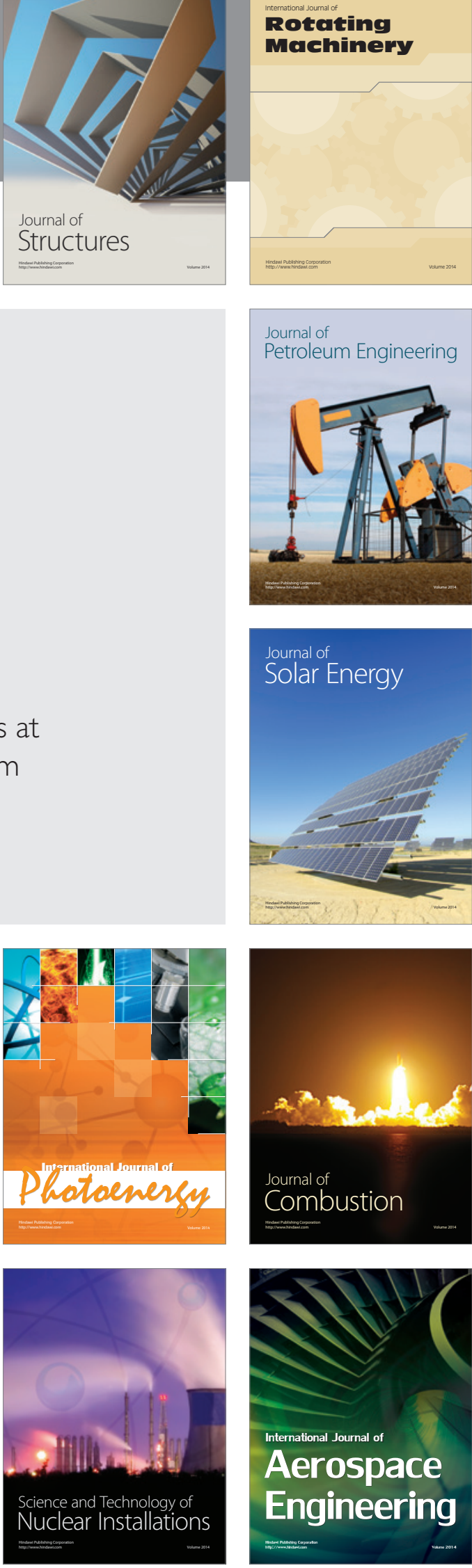